Proceedings of the Edinburgh Mathematical Society (2002) 45, 647-652 (C)

DOI:10.1017/S0013091500001073 Printed in the United Kingdom

\title{
DIAGONALS IN TENSOR PRODUCTS OF OPERATOR ALGEBRAS
}

\author{
VERN I. PAULSEN ${ }^{1}$ AND ROGER R. SMITH ${ }^{2}$ \\ ${ }^{1}$ Department of Mathematics, University of Houston, 4800 Calhoun Road, \\ Houston, TX 77204-3476, USA (vern@math.uh.edu) \\ ${ }^{2}$ Department of Mathematics, Texas A\&M University, College Station, \\ TX 77843-3368, USA (rsmith@math.tamu.edu)
}

(Received 2 November 2000)

\begin{abstract}
In this paper we give a short, direct proof, using only properties of the Haagerup tensor product, that if an operator algebra $A$ possesses a diagonal in the Haagerup tensor product of $A$ with itself, then $A$ must be isomorphic to a finite-dimensional $C^{*}$-algebra. Consequently, for operator algebras, the first Hochschild cohomology group $H^{1}(A, X)=0$ for every bounded, Banach $A$-bimodule $X$, if and only if $A$ is isomorphic to a finite-dimensional $C^{*}$-algebra.
\end{abstract}

Keywords: diagonal; tensor product; Haagerup; cohomology

AMS 2000 Mathematics subject classification: Primary 46L06

Secondary 46L05

\section{Introduction}

Let $A$ be a complex algebra with unit 1. A diagonal in $A \otimes A$ is an element $u=\sum a_{i} \otimes b_{i}$ such that $\sum a_{i} b_{i}=1$ and $\sum\left(a a_{i}\right) \otimes b_{i}=\sum a_{i} \otimes\left(b_{i} a\right)$ for every $a \in A$. For example, if $\mathbb{M}_{n}$ denotes the algebra of $n \times n$ complex matrices and $E_{i j}$ denotes the standard matrix units, then $\sum_{i=1}^{n} E_{i 1} \otimes E_{1 i}$ is easily seen to be a diagonal in $\mathbb{M}_{n} \otimes \mathbb{M}_{n}$.

It is fairly well known that the existence of a diagonal is equivalent to the vanishing of the first Hochschild cohomology $H^{1}(A, X)$ for every $A$-bimodule $X$. Since this fact is elementary, we quickly recall a proof, for clarity. First, assume that we have a diagonal $u$, as above, and that we are given an $A$-bimodule $X$ and a derivation $\delta: A \rightarrow X$. If we set $x=\sum \delta\left(a_{i}\right) b_{i}$, then it is easily checked that $\delta(a)=x a-a x$ and so every derivation into $X$ is inner. That is, $H^{1}(A, X)=0$ for every $X$. To prove the converse, one simply considers the $A$-bimodule $A \otimes A$ and lets $X$ be the submodule which is the kernel of the product map. The map $\delta: A \rightarrow X$ given by $\delta(a)=a \otimes 1-1 \otimes a$ is easily seen to be a derivation. If $w \in X$ is the element that implements this necessarily inner derivation, then it is easily checked that $u=1 \otimes 1-w$ is the desired diagonal.

The above proof easily extends to the case of various topological algebras, where the module actions and derivations are restricted to those which are continuous in some 
appropriate sense. The only change that must be made is that the algebraic tensor product of $A$ with itself is replaced by its completion in some appropriate topology.

In this setting, Helemskii [4] and Selivanov [8] proved that a $C^{*}$-algebra $A$ has the property that every bounded derivation into every bounded $A$-bimodule is inner if and only if $A$ is finite dimensional. By the above remarks this can be seen to be equivalent to characterizing those $C^{*}$-algebras $A$ which possess a diagonal in the projective tensor product of $A$ with itself. The first author extended this result [5] by proving that a $C^{*}-$ algebra $A$ has the property that every completely bounded derivation into every operator $A$-bimodule is inner if and only if $A$ is finite dimensional. Again by the above remarks, this latter result is equivalent to proving that the only $C^{*}$-algebras $A$ which possess a diagonal in the Haagerup tensor product of $A$ with itself are the finite-dimensional $C^{*}$ algebras. Because the projective tensor norm is larger than the Haagerup tensor norm, this latter result implies the result of Helemskii and Selivanov.

Unfortunately, all of the proofs cited above relied on non-trivial results. In particular, the proof in [5] relied on deep results about nuclear and injective $C^{*}$-algebras. The purpose of this paper is to state a more general result, and to give a short, self-contained proof using only properties of the Haagerup tensor product. Specifically, we show that if $A$ is any algebra of operators on a Hilbert space with a diagonal in the Haagerup tensor product of $A$ with itself, then $A$ is necessarily isomorphic to a finite direct sum of matrix algebras.

\section{Main results}

In this section we present our main results. Let $H$ be a Hilbert space and let $B(H)$ denote the algebra of bounded linear operators on $H$. We let $A$ be any subalgebra of $B(H)$ which contains the identity operator. We shall call each such algebra an algebra of operators, and we note that we do not require it to be self-adjoint.

We briefly recall the definition of the Haagerup tensor product. Given $w \in A \otimes A$ we set

$$
\|w\|_{h}=\inf \left\{\left\|\sum a_{i} a_{i}^{*}\right\|^{1 / 2}\left\|\sum b_{i}^{*} b_{i}\right\|^{1 / 2}\right\}
$$

where the infimum is taken over all ways to express $w$ as a finite sum $\sum a_{i} \otimes b_{i}$ of elementary tensors. This quantity defines a norm on $A \otimes A$ called the Haagerup tensor norm and the completion of $A \otimes A$ in this norm is called the Haagerup tensor product of $A$ with itself and is denoted $A \otimes_{h} A$.

This tensor norm has two very nice properties that we shall use. The first is that any $w$ in the completion has a representation as a norm-convergent series, $w=\sum_{i=1}^{\infty} a_{i} \otimes b_{i}$ with $\left\|\sum_{i=1}^{\infty} a_{i} a_{i}^{*}\right\|$ and $\left\|\sum_{i=1}^{\infty} b_{i}^{*} b_{i}\right\|$ both finite. The second is that such a representation may be chosen so that $\left\{a_{i}\right\}_{i=1}^{\infty}$ and $\left\{b_{i}\right\}_{i=1}^{\infty}$ are both strongly independent sets in the following sense. A sequence of elements $\left\{a_{i}\right\}_{i=1}^{\infty}$ which defines a bounded operator $\left(a_{1}, a_{2}, \ldots\right) \in B\left(H^{\infty}, H\right)$ is strongly independent if the equation $\sum_{i=1}^{\infty} \lambda_{i} a_{i}=0$, where $\left\{\lambda_{i}\right\}_{i=1}^{\infty} \in \ell^{2}$, can only be satisfied by $\lambda_{i}=0, i \geqslant 1$. An equivalent formulation $[\mathbf{1}$, 
Lemma 2.2] is that the subspace

$$
\left\{\left(\phi\left(a_{1}\right), \phi\left(a_{2}\right), \ldots\right): \phi \in A^{*}\right\}
$$

is norm dense in $\ell^{2}$. For these facts about the Haagerup tensor product we refer the reader to $[\mathbf{2}, \mathbf{3}, \mathbf{9}, \mathbf{1 0}]$.

Theorem 2.1. Let $A$ be an algebra of operators on a Hilbert space. If there is a diagonal in $A \otimes_{h} A$, then $A$ is finite dimensional.

Proof. Let $u=\sum_{i=1}^{\infty} a_{i} \otimes b_{i}$ be a diagonal, where the series is norm convergent and $\left\{a_{i}\right\}_{i=1}^{\infty}$ and $\left\{b_{i}\right\}_{i=1}^{\infty}$ are strongly independent. Since

$$
\sum_{i=1}^{\infty} a_{i} b_{i}=1
$$

is a norm-convergent series, we may choose $M$ so that

$$
\left\|\sum_{i=1}^{M} a_{i} b_{i}-1\right\|<\frac{1}{2}
$$

and we set $c=\left(\sum_{i=1}^{M} a_{i} b_{i}\right)^{-1}$. From the Neumann series we know that $\|c\|<2$. Now define two constants $k$ and $\varepsilon$ by

$$
k=\max \left\{\left\|\sum_{i=1}^{\infty} a_{i} a_{i}^{*}\right\|^{1 / 2},\left\|\sum_{i=1}^{\infty} b_{i}^{*} b_{i}\right\|^{1 / 2}\right\}, \quad \varepsilon=\left(8 M k^{2}\right)^{-1} .
$$

Since, for each $x \in A$, the series

$$
\sum_{i=1}^{\infty} x a_{i} \otimes b_{i}=\sum_{i=1}^{\infty} a_{i} \otimes b_{i} x
$$

are norm convergent, we may apply, by [3, Proposition 3.7], an element $\phi \in A^{*}$ to (2.5) to obtain

$$
\sum_{i=1}^{\infty} \phi\left(x a_{i}\right) b_{i}=\sum_{i=1}^{\infty} \phi\left(a_{i}\right) b_{i} x .
$$

From the strong independence of $\left\{a_{i}\right\}_{i=1}^{\infty}$, we may choose linear functionals $\phi_{j} \in A^{*}$, $1 \leqslant j \leqslant M$, such that

$$
\left\|\left(\phi_{j}\left(a_{1}\right), \phi_{j}\left(a_{2}\right), \ldots\right)-e_{j}\right\|_{2}<\varepsilon, \quad 1 \leqslant j \leqslant M,
$$

where $\left\{e_{j}\right\}_{j=1}^{\infty}$ denotes the canonical orthonormal basis for $\ell^{2}$.

It now follows that

$$
\left\|b_{j} x-\sum_{i=1}^{\infty} \phi_{j}\left(a_{i}\right) b_{i} x\right\| \leqslant \varepsilon\left\|\sum_{i=1}^{\infty} x^{*} b_{i}^{*} b_{i} x\right\|^{1 / 2} \leqslant \varepsilon k\|x\|,
$$


for $1 \leqslant j \leqslant M$, and for all $x \in A$. Using (2.6), we have that

$$
\left\|b_{j} x-\sum_{i=1}^{\infty} \phi_{j}\left(x a_{i}\right) b_{i}\right\| \leqslant \varepsilon k\|x\|,
$$

for $1 \leqslant j \leqslant M$, and for all $x \in A$.

Since $\lim _{n \rightarrow \infty}\left\|\sum_{i=n}^{\infty} a_{i} a_{i}^{*}\right\|=0$, we may choose $N$ sufficiently large that

$$
\left\|b_{j} x-\sum_{i=1}^{N} \phi_{j}\left(x a_{i}\right) b_{i}\right\| \leqslant 2 \varepsilon k\|x\|
$$

holds for $1 \leqslant j \leqslant M$, and for all $x \in A$. The inequality $\left\|a_{j}\right\| \leqslant k$ follows from (2.4), and so the relation

$$
\left\|\sum_{j=1}^{M}\left[a_{j} b_{j} x-\sum_{i=1}^{N} \phi_{j}\left(x a_{i}\right) a_{j} b_{i}\right]\right\| \leqslant 2 \varepsilon M k^{2}\|x\|
$$

is a consequence of multiplying the expression in (2.10) on the left by $a_{j}$ and summing over $j$. Now multiply (2.11) on the left by $c$ and use (2.4) to obtain

$$
\left\|x-\sum_{j=1}^{M} \sum_{i=1}^{N} \phi_{j}\left(x a_{i}\right) c a_{j} b_{i}\right\| \leqslant 4 \varepsilon M k^{2}\|x\| \leqslant\|x\| / 2, \quad x \in A .
$$

Define a finite-dimensional subspace of $A$ by

$$
B=\operatorname{span}\left\{c a_{j} b_{i}: 1 \leqslant j \leqslant M, 1 \leqslant i \leqslant N\right\} .
$$

The inequality (2.12) implies that the Banach space quotient map from $A$ to $A / B$ has norm at most $\frac{1}{2}$, which can only happen when $A=B$. We conclude that $A$ is finite dimensional.

Ruan [7] has introduced another Hochschild cohomology for operator algebras which uses a family of maps called the jointly completely bounded maps. The relevant tensor norm for this cohomology is called the operator space projective tensor norm.

Corollary 2.2. Let $A$ be an algebra of operators on a Hilbert space. If there is a diagonal in either the projective or operator space projective tensor product of $A$ with itself, then $A$ is finite dimensional.

Proof. For any element in the algebraic tensor product $A \otimes A$, we have that its projective tensor norm is at least as large as its Haagerup norm. Thus, the identity map on $A \otimes A$ extends to a contractive map from the projective tensor product to the Haagerup tensor product. It is easily checked that if $u$ is a diagonal in the projective tensor product, then its image under this map is a diagonal in the Haagerup tensor product, and the result follows from Theorem 2.1. A similar argument applies to the operator space projective tensor product. 
Note that, since any $A$ as above is finite dimensional, the algebraic tensor product is complete in every tensor norm. Thus we are reduced to the purely algebraic problem of determining those finite-dimensional complex algebras $A$ that have a diagonal in $A \otimes A$, which is essentially Burnside's Theorem. We supply a simple proof below that is based on the ideas that we have already introduced.

Theorem 2.3. Let $A$ be a finite-dimensional, unital, complex algebra. If $A$ has a diagonal in $A \otimes A$, then $A$ is isomorphic to a direct sum of matrix algebras.

Proof. Since $A$ can be represented as the algebra of left multiplication operators on itself, we may assume that $A$ is a subalgebra of $\mathbb{M}_{n}$ for some $n$.

Now suppose that $p$ is an invariant orthogonal projection for $A$, that is, $p a p=a p$ for all $a$ in $A$. Then it is easily seen that $X \equiv p \mathbb{M}_{n}(1-p)$ is an $A$-bimodule, and that the equation

$$
\delta(a) \equiv p a(1-p)=p a-a p
$$

defines a derivation of $A$ into $X$. By hypothesis, there exists $x \in X$ such that

$$
\delta(a)=a x-x a, \quad a \in A .
$$

Combining these last two equations, we see that $(p+x)$ commutes with $A$. Since $x^{2}=0$, the element $y \equiv 1+x$ is invertible in $\mathbb{M}_{n}$ with inverse $y^{-1}=1-x$.

The equations

$$
p(1+x) a(1-x)=(p+x) a(1-x)=a(p+x)(1-x)=a p
$$

and

$$
(1+x) a(1-x) p=(1+x) a p=(1+x) \text { pap }=\text { pap }=a p
$$

show that $p$ commutes with $y A y^{-1}$, and thus reduces this algebra.

By inductively choosing such projections $p$ and conjugating by the corresponding invertible elements, we may assume that the representation $\pi: A \rightarrow \mathbb{M}_{n}$ is a finite direct sum of representations, $\pi_{i}: A \rightarrow \mathbb{M}_{n_{i}}, i=1, \ldots, k$, where the image $\pi_{i}(A)$ is a subalgebra of $\mathbb{M}_{n_{i}}$ that has no non-trivial invariant projections. Thus, $\pi_{i}(A)$ is a transitive subalgebra, and hence $\pi_{i}(A)=\mathbb{M}_{n_{i}}$ by Burnside's Theorem [6, Corollary 8.6].

Using the simplicity of each matrix algebra, it is now easy to argue that $A$ is isomorphic to a finite direct sum of matrix algebras. To see this, note that if $J_{i}=\operatorname{ker}\left(\pi_{i}\right)$, then $\pi_{j}\left(J_{i}\right)$ is either $\mathbb{M}_{n_{j}}$ or (0) and argue by induction on $k$.

Corollary 2.4. If $A$ is an algebra of operators and $A$ has a diagonal in one of the Haagerup, projective or operator space projective tensor products of $A$ with itself, then $A$ is isomorphic to a finite direct sum of matrix algebras.

We end by formally stating the equivalent theorems in terms of Hochschild cohomology. If $A$ is any Banach algebra, then by an $A$-bimodule $X$ we mean any Banach space $X$ equipped with an $A$-bimodule action satisfying $\|a x b\| \leqslant c\|a\|\|x\|\|b\|$, for some constant c. An A-derivation is a bounded linear map $\delta: A \rightarrow X$ satisfying $\delta(a b)=a \delta(b)+\delta(a) b$. 
An $A$-derivation is inner if there exists $x$ in $X$ such that $\delta(a)=a x-x a$. Finally, $H^{1}(A, X)$ denotes the quotient of the space of all bounded derivations by the space of inner derivations.

Corollary 2.5. Let $A$ be a Banach algebra, which has a bounded faithful representation as an algebra of operators on a Hilbert space. Then $H^{1}(A, X)=0$ for every bounded $A$-bimodule $X$ if and only if $A$ is isomorphic to a finite direct sum of matrix algebras.

Remark 2.6. Similar results hold for the completely bounded Hochschild cohomology of an operator algebra. For the definitions and results see [5].

Acknowledgements. Both authors were partly supported by grants from the NSF. V.I.P. also thanks the Department of Mathematics, Rice University, where parts of this research were completed.

\section{References}

1. S. D. Allen, A. M. Sinclair and R. R. Smith, The ideal structure of the Haagerup tensor product of $C^{*}$-algebras, J. Reine Angew. Math. 442 (1993), 111-148.

2. D. P. Blecher And V. I. PAulsen, Tensor products of operator spaces, J. Funct. Analysis 99 (1991), 262-292.

3. D. P. Blecher And R. R. Smith, The dual of the Haagerup tensor product, J. Lond. Math. Soc. 45 (1992), 126-144.

4. A. HelemskiI, The homology of Banach and topological algebras (Kluwer, Dordrecht, 1989).

5. V. I. PAulsen, Relative Yoneda cohomology for operator algebras, J. Funct. Analysis 157 (1998), 358-393.

6. H. Radjavi And P. Rosenthal, Invariant subspaces, Ergebnisse der Mathematik und ihrer Grenzgebeite, Band 77 (Springer, 1977).

7. Z.-J. RuAn, Operator amenability of $A(G)$, Am. J. Math. 117 (1995), 1449-1474.

8. Yu. V. Selivanov, Biprojective Banach algebras, Math. USSR Izv. 15 (1980), 387-399.

9. A. M. Sinclair AND R. R. SMITH, Hochschild cohomology of von Neumann algebras, London Mathematical Society Lecture Note Series, no. 203 (Cambridge University Press, 1995).

10. R. R. Smith, Completely bounded module maps and the Haagerup tensor product, $J$. Funct. Analysis 102 (1991), 156-175. 